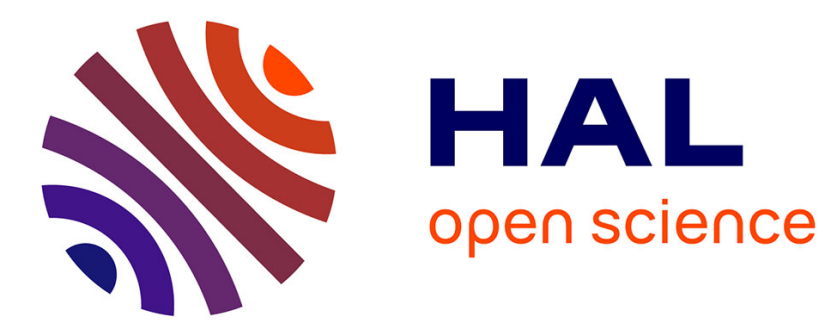

\title{
Spectromètre interférentiel à sélection par l'amplitude de modulation \\ Pierre Connes
}

\section{To cite this version:}

Pierre Connes. Spectromètre interférentiel à sélection par l'amplitude de modulation. Journal de Physique et le Radium, 1958, 19 (3), pp.215-222. 10.1051/jphysrad:01958001903021500 . jpa00235803

\section{HAL Id: jpa-00235803 https://hal.science/jpa-00235803}

Submitted on 1 Jan 1958

HAL is a multi-disciplinary open access archive for the deposit and dissemination of scientific research documents, whether they are published or not. The documents may come from teaching and research institutions in France or abroad, or from public or private research centers.
L'archive ouverte pluridisciplinaire HAL, est destinée au dépôt et à la diffusion de documents scientifiques de niveau recherche, publiés ou non, émanant des établissements d'enseignement et de recherche français ou étrangers, des laboratoires publics ou privés. 


\title{
SPECTROMETRE INTERFÉRENTIEL A SÉLECTION PAR L'AMPLITUDE DE MODULATION (1)
}

\author{
Par P. CONNES, \\ Laboratoire de Bellevue.
}

\begin{abstract}
Résumé. - Description d'une nouvelle méthode spectrométrique spécialement adaptée à l'infrarouge. En remplaçant les deux miroirs d'un interféromètre de Michelson par des réseaux utilisés sous la même incidence, et en faisant varier linéairement en fonction du temps la différence de marche entre les deux faisceaux on peut moduler dans un spectre complexe un seul élément spectral, de largeur égale à la limite de résolution théorique du réseau. L'angle solide du faisceau utilisable étant beaucoup plus grand que dans l'emploi classique du réseau, cette méthode permet un gain de luminosité important. Le premier appareil réalisé est décrit ainsi que les premiers résultats obtenus.
\end{abstract}

\begin{abstract}
A new spectrometric method, chiefly useful in the infrared is described. By replacing the mirrors of a Michelson interferometer by two gratings under equal incidence and varying the path difference between the two beams in a linear way, one is able to modulate in a complex spectrum a single element whose width is equal to the theoretical resolution limit of the grating. The solid angle of the admissible beam is much greater than in a classical spectrometer, thus this method gives an important increase in luminous flux. The first instrument is described together with the first experimental results.
\end{abstract}

Deux méthodes différentes permettant d'augmenter l'énergie disponible en spectrométrie infrarouge sont examinées au cours du Colloque. Suivant la première (employée par de nombreux participants, mais dont l'intérêt est plus particulièrement illustré par les communications de R. Chabbal et R. Greenler) on augmente l'étendue du faisceau accepté par le spectromètre, en remplaçant un appareil à réseau par un ou plusieurs étalons FabryPerot en série. La seconde (qui pourrait être baptisée Spectrométrie interférentielle à sélection par la fréquence de modulation) est décrite dans les communications de P. Fellgett, J. Strong et G. A. Vanasse, J. Connes, H. A. Gebbie et L. Mertz; elle augmente le temps disponible pour la mesure de chaque élément'spectral en permettant l'étude simultanée des différents éléments spectraux $\left(^{2}\right)$.

Le spectromètre interférentiel à sélection par l'amplitude de modulation (SISAM) que nous allons décrire ici se rattache sans ambiguité possible au premier type (augmentation de l'étendue), bien que par les moyens employés, il rappelle un peu le second. Il comporte un nouveau mode d'utilisation des appareils dispersifs (réseaux ou prismes) qui permet d'obtenir leur pouvoir de résolution théorique, tout en acceptant un faisceau de même angle solide qu'un étalon F.-P. de même résolution.

Nous nous contenterons d'en indiquer le prin-

(1) Les deux communications de P. Connes ont ici été rassemblées en un seul article.

(2) Si le modulateur utilisé est un interféromètre de Michelson, l'étendue est la même qu'avec un étalon F.-P., de sorte que les avantages des deux méthodes se trouvent réunis. cipe et les principales propriétés dans le cas de l'application au réseau, renvoyant le lecteur pour une étude plus complète à un article [1] non encore paru au moment du Colloque. Nous décrirons ensuite un peu plus en détails le premier appareil construit et ses performances.

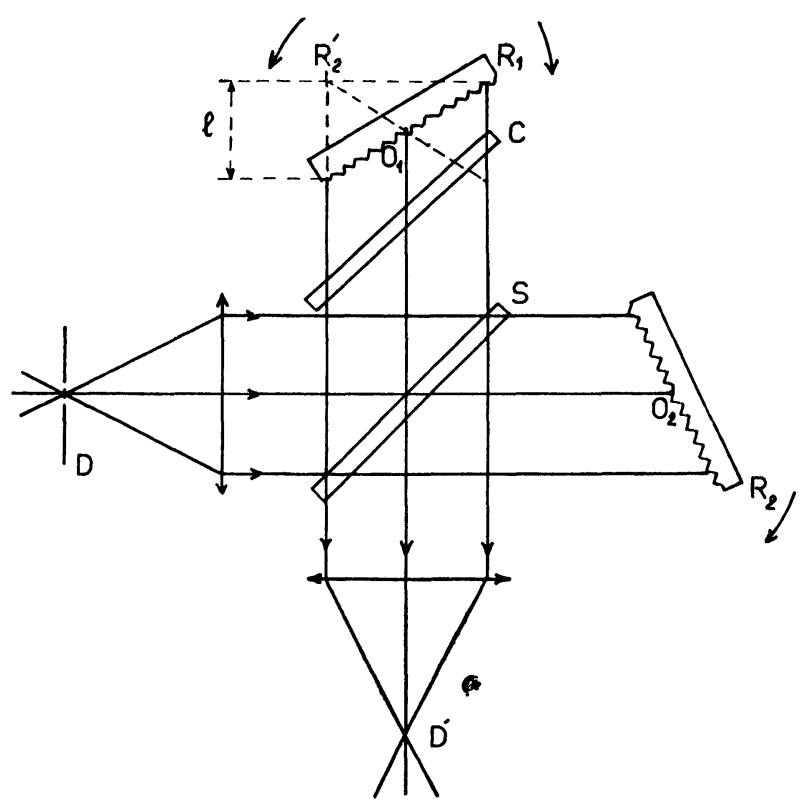

Fig. 1.

1. Principe. - On remplace les deux miroirs d'un interféromètre de Michelson par deux réseaux identiques $R_{1}, R_{2}$ (fig. 1). L'image $R_{2}^{\prime}$ de $R_{2}$ 
coupe $R_{1}$ en son milieu. Si l'appareil est éclairé par un faisceau parallèle monochromatique de nombre d'onde $\sigma_{0}$ et si l'angle d'incidence est tel que les rayons soient renvoyés exactement sur eux-mêmes (comme dans un montage Littrow) les deux faisceaux sortants sont parallèles, comme avec un interféromètre de Michelson classique dont les miroirs (réel et virtuel) sont parallèles et reçoivent un faisceau parallèle sous l'incidence normale. Dans les deux cas l'état d'interférence est le même pour tous les rayons. Si l'on fait varier la différence de marche $\delta$ entre les deux faisceaux (par exemple en faisant tourner la lame compensatrice $\mathrm{C}$ ), le flux lumineux transporté par le faisceau sortant est modulé à $100 \%$ avec une fréquence $N=v \sigma_{9}$ (l'énergie étant alternativement transmise et réfléchie par l'interféromètre).

Si maintenant on fait tourner $R_{1}$ et $R_{2}$ d'un petit angle, dans le même sens $\left(R_{1}\right.$ et $R_{2}^{\prime}$ tournant alors en sens inverse), tout se passe comme si l'on déréglait un interféromètre de Michelson classique et la profondeur de modulation baisse rapidement pour la radiation $\sigma_{0}$; en revanche elle augmente et devient égale à l'unité pour une autre radiation $\sigma$, voisine de $\sigma_{0}$ et telle que ses rayons soient maintenant renvoyés sur eux-mêmes. Il est donc possible de faire l'enregistrement d'un spectre complexe en l'explorant par rotation des réseaux (comme avec un spectromètre classique) à condition d'utiliser un amplificateur qui transmette seulement la composante alternative du signal lumineux reçu par le détecteur.

Il est facile de calculer la forme du pic de modulation obtenu au passage d'une raie infiniment fine, c'est-à-dire celle de la fonction d'appareil. A condition de supposer les réseaux diaphragmés par des losanges dont les diagonales soient égales à leur hauteur et leur largeur, on trouve que cette fonction est la même que pour un spectromètre Littrow équipé d'un réseau identique (non diaphragmé) et dont les fentes sont infiniment fines, c'est-à-dire une fonction de diffraction $(\sin x / x)^{2}$. Le pouvoir de résolution est donc égal au pouvoir de résolution théorique classique du réseau $\mathcal{R}_{0}=2 l / \lambda$ ( $l$ étant la projection de la largeur du réseau sur la direction des rayons lumineux).

Supposons maintenant que le diaphragme d'entrée $D$ au lieu d'être infiniment petit admette des rayons inclinés d'un angle $\varepsilon$ par rapport aux précédents. La différence de marche entre deux rayons émergents issus d'un même incident dépend de $\varepsilon$; la variation est maximum pour un rayon tombant sur le bord des réseaux, où elle est la même qu'avec un interféromètre de Michelson classique dont les miroirs seraient parallèles et distants de $l$. On en déduit que $D$ doit être circulaire, 'et que l'angle solide $\Omega$ admissible est le même qu'avec un interféromètre de Michelson (ou un étalon F.-P.) donnant le même pouvoir de réso- lution. On sait [2] qu'avec ces appareils le compromis le plus satisfaisant entre résolution et luminosité est obtenu en adoptant $\Omega$ tel que :

$$
\Omega R_{0}=2 \pi
$$

et que, dans ce cas, le pouvoir de résolution réel est $\mathcal{R}=0,8 \mathcal{R}_{0}$. Il n'est d'ailleurs pas possible de s'écarter beaucoup de cette condition avec le SISAM qui est pratiquement un appareil à résolution fixe. Pour avoir différents $\mathcal{R}_{0}$, il est nécessaire d'utiliser des réseaux d'angles de blaze différents.

Le gain d'étendue $G$ par rapport au spectromètre Littrow utilisant le même réseau à la même résolution, et des fentes de hauteur angulaire $\beta$ est, compte tenu de la perte de la moitié de la surface du réseau causée par le diaphragme losange :

$$
G=\frac{\pi}{2 \beta \operatorname{tg} i_{0}} .
$$

Le SISAM possède une autre propriété, secondaire mais importante : la fréquence de modulation étant proportionnelle à la fréquence lumineuse n'est pas la même pour les différentes radiations que le réseau renvoie superposées dans des ordres différents; on peut ainsi les séparer par filtrage électrique, comme dans les dispositifs à modulation interférentielle décrits par G. Strong [3]. Il est ainsi possible soit de conserver un seul ordre pour explorer une petite région spectrale à haute résolution (ce qui paraît devoir être l'emploi essentiel de la méthode), soit d'enregistrer plusieurs ordres simultanément au moyen d'un amplificateur à plusieurs canaux parallèles accordés sur des fréquences proportionnelles aux numéros des ordres. Le gain de rapidité ainsi obtenu rappelle, tout en étant beaucoup plus limité, celui que procure la méthode par transformation de Fourier.

La réalisation de la modulation interférentielle du SISAM appelle une remarque. Il est impossible de faire varier la différence de marche d'une façon continue; on doit donc se contenter d'une variation en dents de scie symétriques obtenue par un mouvement de rotation de la compensatrice alternativement linéaire dans un sens et dans l'autre. Le signal modulé sera donc constitué de trains de $n$ périodes sans cohérence entre eux, ce qui ne soulève pas de difficultés pratiques à condition que $n$ soit très supérieur au facteur de surtension $Q$ du circuit sélectif utilisé. Cette condition est toujours facile à satisfaire; d'ailleurs l'emploi de la détection synchrone (le signal de référence devant être produit par l'interféromètre lui-même) permet toujours de réduire la bande de fréquences utilisée autant qu'on le désire.

Le principe du SISAM paraît surtout applicable à l'infra-rouge. En effet le détecteur re- 
çoit à chaque instant non seulement l'énergie " utile » de l'élément spectral modulé mais encore celle des éléments spectraux voisins ( ${ }^{3}$ ). A condition de munir l'interféromètre d'un diaphragme de sortie coincidant avec l'image $\mathrm{D}^{\prime} \mathrm{du}$ diaphragme d'entrée, le rapport énergie parasite non modulée sur énergie utile modulée est peu différent de $\sqrt{\mathcal{R}_{0}}$, si le spectre est continu et uniforme au voisinage de l'élément spectral modulé. Il s'ensuit une augmentation très importante du bruit lorsque celui-ci est dû uniquement au rayonnement, c'est-à-dire lorsque le bruit propre du récepteur (photomultiplicateur refroidi) est négligeable.

Le gain de rapidité est alors très inférieur au gain de luminosité ; il ne peut rester intéressant que dans le cas de spectres peu denses (spectres Raman par exemple).

2. Tolérances optiques et mécaniques. - Les premières ayant déjà été traitées [1] nous ne ferons que rappeler les conclusions. Le SISAM ne pouvant être employé qu'au voisinage de la résolution théorique, il est nécessaire que les deux surfaces d'onde interférentes soient proches de la perfection théorique, c'est-à-dire que les défauts des différentes pièces optiques soient petits par rapport à la longueur d'onde, exigence qui ne paraît pas trop difficile à satisfaire dans l'infra-rouge.

Nous nous arrêterons plus longuement sur les conditions mécaniques auxquelles doit satisfaire le montage.

Dans l'appareil idéal représenté par la figure 1 (dont nous supposerons le plan horizontal) les plans des quatre pièces optiques sont verticaux, ainsi que les traits des réseaux, et l'axe de rotation de chaque réseau coïncide avec un trait. En pratique il suffit qu'au cours de l'exploration du spectre la direction des traits des réseaux reste parfaitement constante. Il faut donc que le parallélisme des traits et de l'axe de rotation soit assuré à une fraction de longueur d'onde près et que l'axe lui-même soit défini avec une précision équivalente. S'il est matérialisé par une surface cylindrique, celle-ci doit avoir les qualités d'une surface optique. Une petite déformation de l'axe se traduit par un déréglage en cours d'enregistrement, les faisceaux diffractés par les réseaux n'étant plus horizontaux. Mais il est facile de les ramener à être parallèles entre eux à la sortie de l'interfomètre, ce qui est pratiquement suffisant, par une petite rotation de la séparatrice autour d'un axe horizontal. Ce réglage permet également de compenser un léger défaut de parallélisme des axes de rotation. Il est donc seulement nécessaire de prévoir deux réglages fins pour l'orientation de chaque réseau dans son boîtier, et un

(3) Et non pas celle de tous les éléments spectraux comme c'est le cas dans la méthode par transformation de Fourier qui ne comporte pas l'emploi d'une dispersion. pour celle de la séparatrice; la verticalité des axes de rotation est assurée par construction avec une précision suffisante. Tous les réglages précédents sont facilement contrôlables par l'observation de franges analogues à celles du coin d'air, visibles sur le plan normal au faisceau passant par $\mathrm{O}_{1}$ lorsque l'interféromètre est éclairé en lumière parallèle monochromatique.

La régularité de la rotation ne pose pas de problèmes particuliers, les exigences sont les mêmes que pour le spectromètre classique équivalent. Les irrégularités du mouvement se traduisent par des déformations de l'échelle des fréquences dans le spectre enregistré et non par des variations d'intensité. L'égalité des angles d'incidence sur les deux réseaux n'a besoin d'être réalisé qu'à une fraction du diamètre angulaire de $D$ près, ce qui est facile. La position relative des deux réseaux et de la lame séparatrice doit être assurée avec la même stabilité que dans tout interféromètre de Michelson. $\mathrm{De}$ même seule la'direction de la compensatrice intervient. Son support peut donc être indépendant de celui des trois autres pièces, ce qui est utile car il reçoit une percussion importante à l'instant où le sens de rotation de la compensatrice s'inverse et pourrait leur transmettre des vibrations.

La variation de la différence de marche n'est pas linéaire en fonction de la rotation de la compensatrice. Il faudra donc soit limiter l'amplitude du mouvement en dents de scie à une valeur très faible ce qui pourrait conduire à un nombre $n$ de périodes cohérentes insuffisant, soit introduire dans le mouvement une légère accélération de sens et de grandeur constants qui compensera les termes du second ordre ce qui est suffisant.

Toute irrégularité dans le mouvement de la compensatrice se traduit par une fluctuation de la fréquence de modulation qui est sans effet sur le pouvoir de résolution, mais cause une fluctuation de l'amplitude du signal modulé après traversée d'un circuit sélectif, c'est-à-dire une sorte de bruit supplémentaire. Ce "bruit " ne possède pas un spectre de fréquence uniforme ; la plus basse fréquence (la plus difficile à filtrer) est $N_{0}$, celle du mouvement en dents de scie lui-même. En conséquence il est préférable de ne pas choisir $N_{0}$ trop basse, c'est-à-dire (la fréquence de modulation $N$ étant imposée, entre certaines limites, par le détecteur), une valeur de $n$ inutilement grande.

Les effets de ce " bruit » supplémentaire peuvent toujours être annulés en donnant à l'enregistreur une constante de temps $\tau$ nettement supérieure à $T_{0}=1 / N_{0}$. Il est donc relativement plus facile d'obtenir de bons résultats lorsqu'on cherche à exploiter le gain de luminosité attendu de la méthode par un accroissement de résolution (ou l'étude d'une source plus faible) à vitesse d'exploration donnée, que par un accroissement de vitesse à résolution donnée. 
3. Description du premier appareil. - Il a été construit en vue de vérifier les principes que nous venons d'exposer, et emploie des réseaux de dimensions assez modestes $(65 \times 76 \mathrm{~mm}, 600$ traits $/ \mathrm{mm}$, angle de blaze $30^{\circ}$ ). Les lames séparatrice et compensatrice sont en verre ; l'appareil est donc utilisable dans le visible et l'infra-rouge proche jusqu'à $2,8 \mu$ environ.

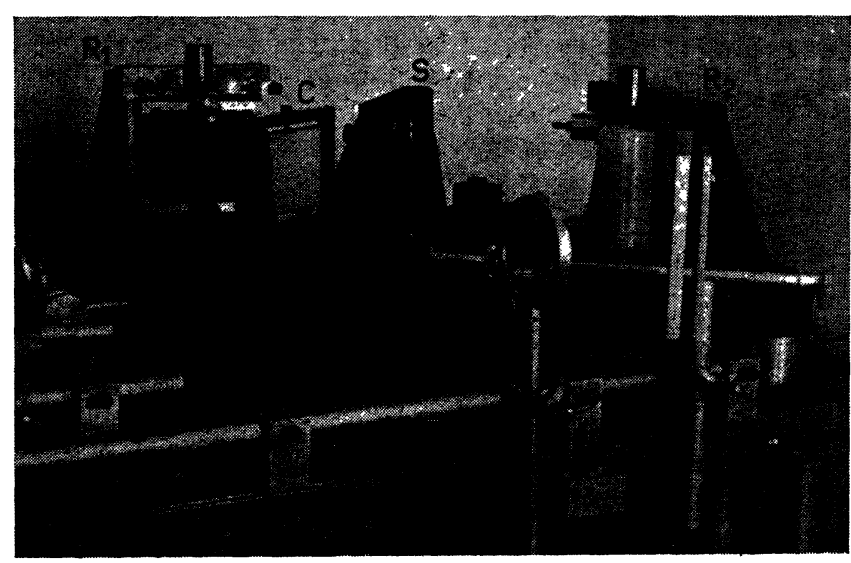

FIG. 2.
Les deux réseaux et la séparatrice sont portés par des consoles de fonte ( $f$ g. 2) placées sur une platine d'acier de $50 \times 50 \mathrm{~cm}$, dont la face inférieure porte le dispositif d'entraînement des réseaux. L'axe de la compensatrice traverse librement cette platine et son support est monté de façon complètement indépendante.

Les boîtiers des réseaux sont munis de deux tourillons cylindriques coaxiaux que des ressorts appliquent contre des "vés " horizontaux ; le poids de l'ensemble est supporté par une bille d'acier sur l'axe des tourillons. La précision de ce dispositif est suffisante pour permettre d'explorer sans déréglage quelques centaines de $\mathrm{cm}^{-1}$ vers $\lambda=1,5 \mu$. Lorsque l'on change de région du spectre, il y a lieu de retoucher le réglage de la séparatrice.

L'entraînement des réseaux ( $f g .3$ ) comporte un moteur synchrone à réducteur, une boîte de vitesses et une vis micrométrique $\mathrm{V}$ appuyant normalement à l'extrémité d'un levier $\mathrm{L}$ de $40 \mathrm{~cm}$ de longueur. L'axe de rotation A du levier, monté sur roulements, porte une poulie $\mathrm{P}$ de $20 \mathrm{~cm}$ de diamètre à deux gorges plates, qui entraîne par l'intermédiaire de deux rubans d'acier $R_{1}, R_{2}$, deux poulies identiques $\mathrm{P}_{1}, \mathrm{P}_{2}$ calées sur les axes des réseaux. La tension des rubans moteurs est assurée par des

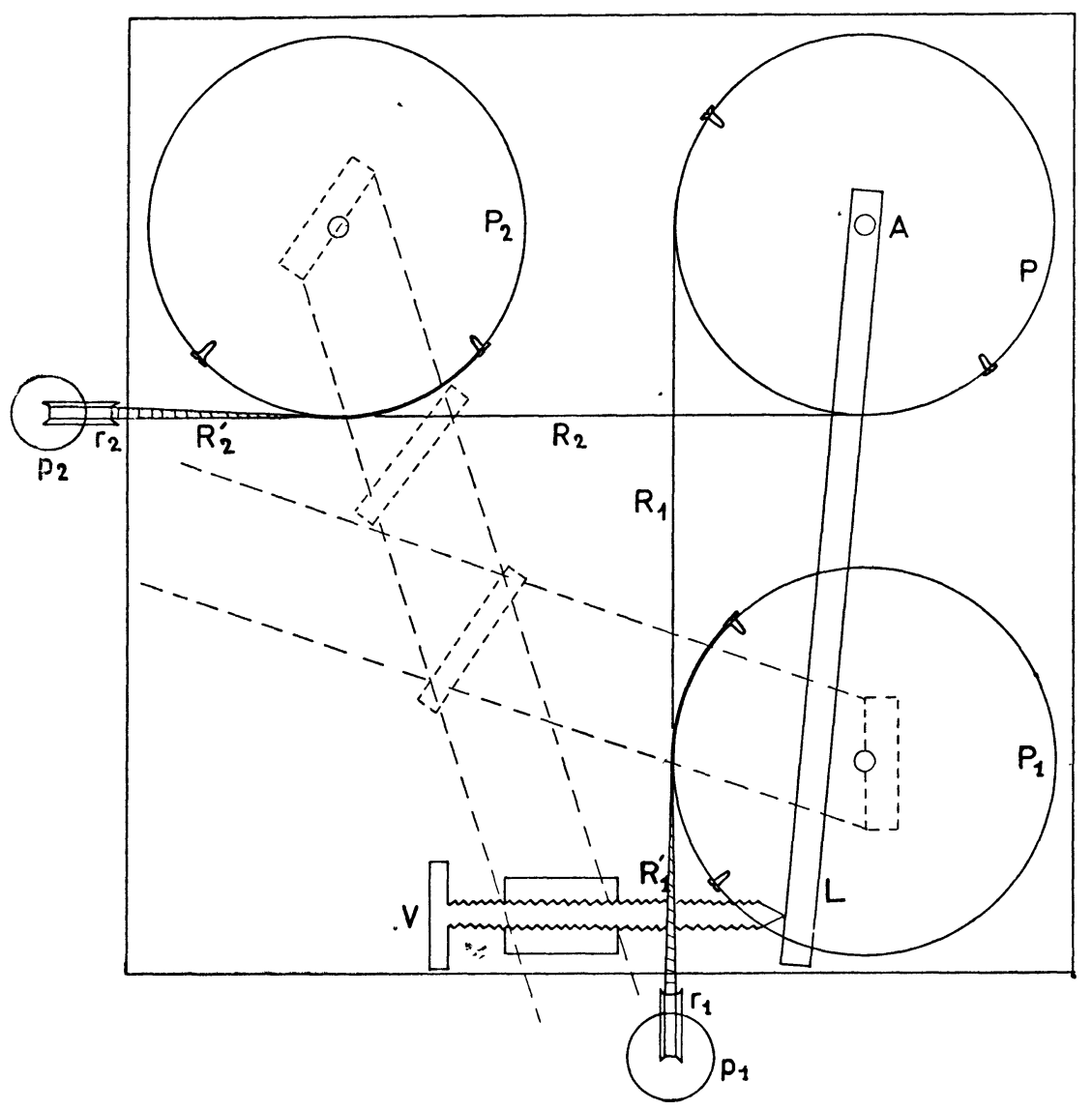

FIG. 3. 
rubans $R_{1}^{\prime}, R_{2}^{\prime}$, parallèles à $R_{1}, R_{2}$, et tendus eux mêmes par des poids $p_{1}, p_{2}$ après passage sur des poulies de renvoi $r_{1}, r_{2}$. Le dispositif d'entraînement n'exerce ainsi presque aucun effort sur l'axe du réseau.

Un premier dispositif d'entraînement de la compensatrice utilisait une came en double spirale d'Archimède portée par un moteur synchrone à réducteur, sur laquelle appuyait un levier calé sur l'axe de la compensatrice. Le mouvement ainsi obtenu n'était jamais exempt de vibrations et la stabilité de la fréquence de modulation était assez médiocre. Un dispositif différent est maintenant employé (fig. 4). L'axe de la compensatrice porte un

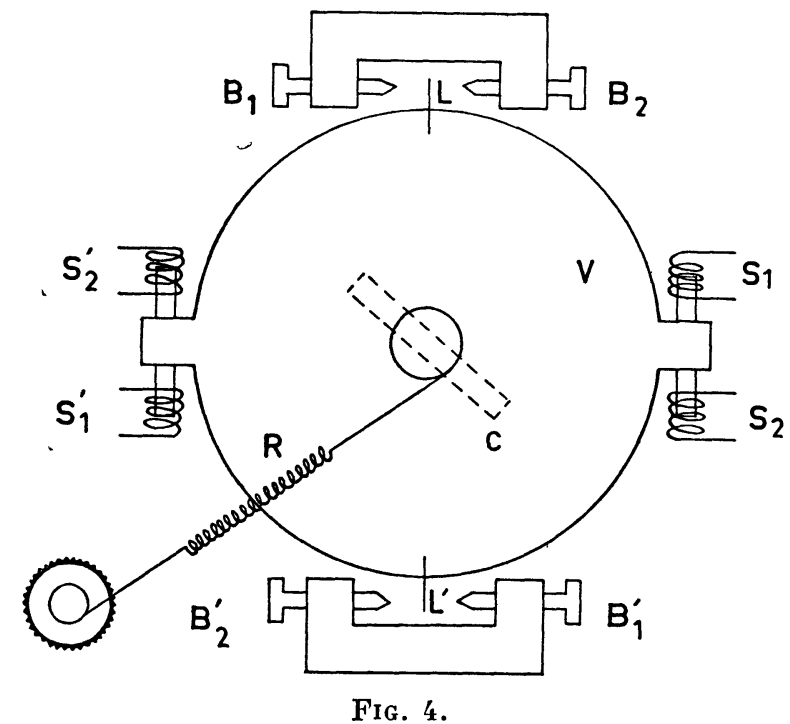

volant $\mathrm{V}$ assez lourd qui peut tourner sans couple de rappel et avec des frottements aussi réduits que possible (son poids est supporté par un pivot à bille de très petites dimensions). Ce volant porte deux lames de ressort plates $L$ et $L^{\prime}$ dont la course est limitée par 4 butées réglables $B_{1}, B_{2}$ et $B_{1}^{\prime}, B_{2}^{\prime}$. Le mouvement est linéaire entre deux chocs ; la durée des chocs, déterminée par le moment d'inertie de V et la raideur des ressorts est inférieure à $1 / 100 \mathrm{~s}$. Afin d'assurer l'entretien du mouvement, le contact des lames $L$ et $L^{\prime}$ sur les butées provoque la décharge de condensateurs à travers des thyratrons dans les bobines $S_{1}, S_{1}^{\prime}$ et $S_{2} S_{2}^{\prime}$ alternativement. La fréquence $N_{0}$ est déterminée par les quantités d'énergie apportée et emportée à chaque période. On obtient une stabilité à long terme suffisante (de l'ordre de $2 \%$ après une période de mise en route) en chargeant les condensateurs à travers des pentodes à courant constant à partir d'une source de haute tension stabilisée, un allongement de la période provoquant une augmentation de la charge du condensateur, donc de l'impulsion d'entretien.

L'amplitude du mouvement est de l'ordre de 1/10 rad et l'accélération nécessaire est obtenue en appliquant par un ressort $R$ un couple de rappel très léger et pratiquement constant. Il est possible avec ce dispositif de faire défiler les franges d'interférences assez régulièrement pour observer sur un oscilloscope des figures de Lissajous presque parfaitement stables (avec $N=160 \mathrm{~Hz}, N_{0}=1 \mathrm{~Hz}$ ), en dehors des variations brusques de phase qui se produisent à l'instant des chocs.

4. Résultats. - Les premiers ont déjà été décrits [1]. L'appareil avait permis d'atteindre dans la région violette du spectre visible un pouvoir de résolution de 95000 , la valeur calculée étant 115000 , et dans l'infra-rouge à $1,6 \mu$ une limite de résolution de $0,2 \mathrm{~cm}^{-1}$ pratiquement égale à la valeur prévue

L'amélioration du dispositif d'entraînement de la compensatrice a maintenant permis d'obtenir des enregistrements sur lesquels les fluctuations sont dues uniquement au bruit du récepteur et non aux irrégularités de la modulation. Nous avons donc cherché à vérifier en enregistrant un spectre d'absorption successivement avec le SISAM et un spectromètre classique (équipé de l'un des réseaux du SISAM) à la même résolution, avec une même source, une même cellule et le même rapport signal/ bruit, si le gain de luminosité $G$ donné par (2) se traduisait bien, comme on pouvait s'y attendre, par un gain $G^{2}$ sur la rapidité $\left(^{4}\right)$.

La résolution théorique des réseaux dans le premier ordre était $\mathcal{R}_{0}=45000$. On pouvart espérer atteindre $\mathcal{R}=0,8 \mathcal{R}_{0}=36000$, en se plaçant dans les conditions définies par (1) avec le SISAM, ou en utilisant des fentes voisines de la largeur à mi-hauteur de la tache centrale de la figure de diffraction avec le spectromètre classique. La limite de résolution correspondante était de $0,17 \mathrm{~cm}^{-1}$.

a) Avec le spectromètre classique, nous avons constaté qu'aucun gain de résolution n'était plus obtenu en prenant des fentes de largeur inférieure à 1,6 fois la figure de diffraction, et on n'a pu dépasser une limite de résolution de $0,25 \mathrm{~cm}^{-1}$. L'écart paraît dû essentiellement aux défauts des

(4) La comparaison a été faite avec la même cellule afin qu'elle soit aussi simple et directe que possible. Si l'on avait adopté les meilleures conditions (envisagées par P. Jacquinot dans sa communication) pour les deux instruments, une cellule de surface $G$ fois plus faible, donnant $\sqrt{G}$ fois moins' de bruit aurait théoriquement pu être employée avec le spectromètre classique et le gain de rapidité du SISAM aurait été $G$ et non $G^{2}$. En réalité une telle cellule n'existe pas ; si l'on s'était placé dans les meilleures conditions pratiques, en utilisant la plus petite cellule possible avec le spectromètre classique le gain obtenu aurait été intermédiaire entre $G$ et $G^{2}$. Nous n'avons pas fait cette expérience, inutile pour notre comparaison. Il faut seulement remarquer que l'enregistrement de la figure 5 (en haut) ne représente pas le meilleur résultat que l'on puisse obtenir avec un spectromètre classique muni de réseaux $65 \times 76 \mathrm{~mm}$. 
fentes $\left({ }^{5}\right)$. L'enregistrement, présenté sur la figure 5 a été obtenu avec une cellule refroidie à la neige carbonique, en utilisant la détection synchrone, à la vitesse de $1 \mathrm{~cm}^{-1}$ en 6 minutes.

Spectromètre Elbert-Fastie.

$f=110 \mathrm{~cm}$

Fentes: $\alpha=3,610^{-5}$ rd $(u=1,6) \beta=1 / 30 \mathrm{rd}$.

Cellule refroidie par $\mathrm{CO}_{2}$ solide (gain II).

Détection synchrone $F=125 \mathrm{~Hz} \tau=15 \mathrm{~s}$.

Exploration $1 \mathrm{~cm}^{-1} / 6 \mathrm{~min}$.

$d \sigma=0.25 \mathrm{~cm}^{-1}$

nterféromètre d̀ réseaux.

Diaphragme losange.

Diaphragme de sortie $\alpha=1,3 \quad 10^{-2} \mathrm{rd}$.

Gain théorique 80 .

Cellule non refroidie.

Détection classique $F=160 \mathrm{~Hz} \quad \Delta F=400 \mathrm{~Hz}$.

Exploration $1 \mathrm{~cm}^{-1} / 10 \mathrm{~s}$.

$d \sigma=0,18 \mathrm{~cm}^{-1}$

b) Avec le SISAM on a obtenu sans aucune difficulté une limite de résolution de $0,18 \mathrm{~cm}^{-1}$ (largeur mesurée à mi-hauteur des raies d'absorption les plus fines). D'autre part le gain de luminosité attendu
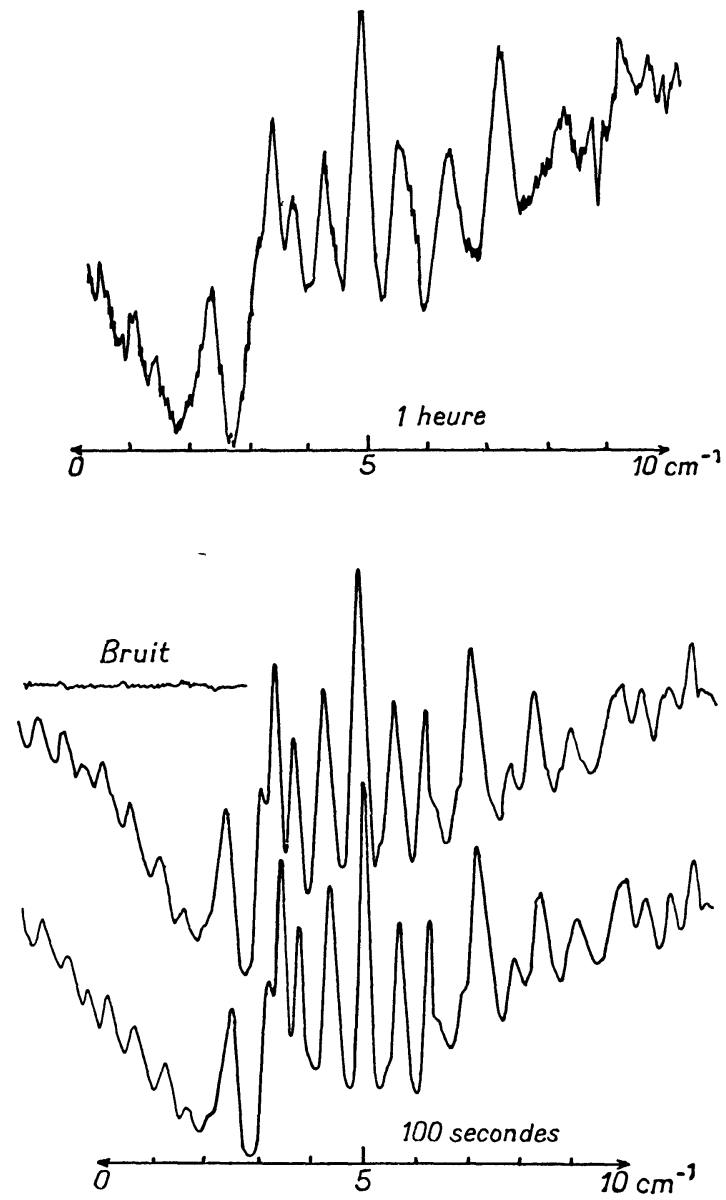

FIG. 5.

Spectre d'absorption de $\mathrm{ICH}_{3}$ à $6050 \mathrm{~cm}^{-1}(1,65 \mu)$

Pression $15 \mathrm{~cm} \mathrm{Hg}$; épaisseur $40 \mathrm{~cm}$

Lampe à incandescence $6 \mathrm{~V}, 5 \mathrm{~A}$. Cellule au $\mathrm{PbS}$

Réseaux : $65 \times 76 \mathrm{~mm} ; 600$ traits $/ \mathrm{mm} \quad \varphi=30^{\circ} 1^{\mathrm{er}}$ ordre

$\mathcal{R}_{0}=46.000 \mathcal{R}=0,8 \mathcal{R}_{0}=36.000 \mathrm{~d} \sigma=0,17 \mathrm{~cm}^{-1}$

était, d'après (2), $G=80$, équivalent à un gain de rapidité de $80^{2}=6400$. Comme il était impossible (à cause du temps de réponse de l'enregistreur) de faire un enregistrement 6400 fois plus rapide que le premier, on a consenti une perte d'un facteur 11 sur la sensibilité de la cellule (équivalent à $11^{2}=121$ sur la vitesse, le bruit n'étant pas modifié) en ne la ręfroidissant pas. L'enregistrement aurait donc $\mathrm{pu}$, en principe, être fait $6400 / 121=53$ fois plus vite que dans le premier cas ; mais comme le dispositif d'entraînement des réseaux ne permettait pas d'obtenir n'importe quelle vitesse d'exploration, l'enregistrement de la

(5) Le spectromètre avait été construit pour servir de monochromateur à un étalon F.-P. et non pour être utilisé au voisinage de la résolution théorique. figure 5 (en bas) a été fait à raison de $1 \mathrm{~cm}^{-1}$ en $10 \mathrm{~s}$, soit 36 fois plus vite que le précédent, et le rapport signal/bruit s'est trouvé sensiblement meilleur sur ce deuxième enregistrement, ainsi que l'on pouvait s'y attendre. Deux traces successives sont présentées afin de montrer la reproductibilité (qui n'est limitée que par le bruit) ainsi qu'une trace obtenue en arrêtant l'exploration afin de permettre l'estimation du bruit.

La figure 6 représente deux enregistrements successifs d'une portion plus étendue du même spectre obtenus avec le SISAM à une vitessse double $\left(1 \mathrm{~cm}^{-1}\right.$ en $\left.5 \mathrm{~s}\right)$, et toujours sans refroidir la cellule. La largeur à mi-hauteur d'une raie est explorée en $1 \mathrm{~s}$.

Pour ces enregistrements la détection classique 
était utilisée avec une large bande passante ; il n'y avait en effet pas d'ordres parasites à éliminer et d'autre part le signal était très supérieur au bruit. Mais la détection synchrone a également fonctionné de façon satisfaisante ; le signal de référence était fourni par une deuxième cellule recevant un faisceau issu de la même source, modulé par l'interféromètre et ne traversant pas la cuve à absorption.
A la suite de ces premiers essais qui montrent seulement le gain de rapidité que l'on peut attendre du SISAM, nous avons entrepris la construction d'interféromètres munis des plus grands réseaux existant actuellement, ainsi que de lames transparentes à l'infra-rouge moyen. Le gain de luminosité se traduira alors par une amélioration des limites de résolution.

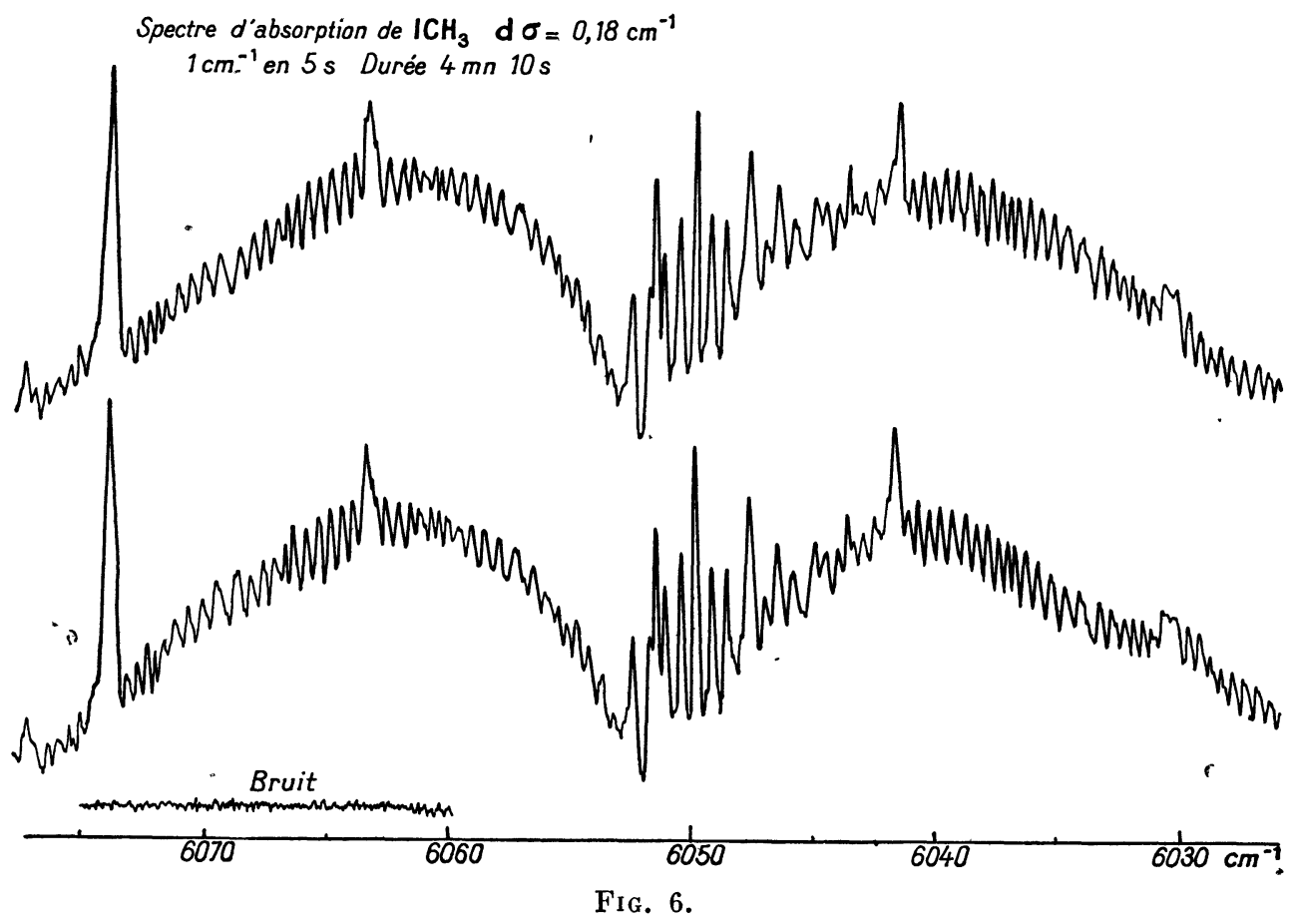

\section{BIBLIOGRAPHIE}

[1] Connes (P.), Optica Acta, 1957, 4, 136.

[2] JACQuinot (P.), J. Opt. Soc. Amer., 1954, 44, 761.

[3] Strong (J.),J.Opt. Soc. Amer., 1957, 47, 354.

\section{DISCUSSIONS}

P. Fellgett. - J'aimerais savoir d'où vient l'idée initiale du SISAM ?

P. Connes. - Nous cherchions à imaginer un monochromateur dont l'étendue s'adapte bien à celle d'un étalon de Fabry-Perot, c'est-à-dire possédant la symétrie de révolution. Nous avons envisagé différents systèmes possédant cette symétrie (dispositifs à isolement focal à lentilles ou à réseaux circulaires ou cylindriques) sans leur trouver sur les appareils à fentes la supériorité d'étendue que possède l'interféromètre de Fabry-Perot; nous avons alors compris que cette supériorité est due à la présence de lames semi-transparentes et ne pouvant construire facilement des réseaux semitransparents, nous avons pensé à utiliser une image virtuelle d'un réseau, donc un interféromètre de Michelson.

A. Maréchal. - Exxiste-t-il un rapport entre la vitesse de rotation des réseaux et celle de défilement des franges?

$P$. Connes. - Il n'y a pas de relation déterminée ; il est seulement nécessaire que, pendant que la réseau explore un élément spectral, il défile assez de franges pour que la fréquence de modulation soit définje.

P. Fellgett. - N'a-t-on pas les relations habituelles pour l'intensité des "ghosts"?

$P$. Connes. - Hélas, non ! c'est un vice caché de la méthode. Dans le SISAM tel que je l'ai décrit, les "ghosts " ont pour intensité relative la racine carrée de leur intensité relative dans la méthode classique. On peut corriger ce défaut soit en utilisant un diaphragme à bords découpés selon une fonction périodique, soit en employant les réseaux avec leurs 
traits perpendiculaires et non parallèles. Ceci ne sera probablement pas nécessaire dans l'infra-rouge où la perfection des réseaux est relativement plus grande.

G. Stroke. - Les réseaux réalisés au M. I. T. sous la direction de G. R. Harisson atteignent 10 pouces $(254 \mathrm{~mm})$ de largeur, avec des déviations moyennes de la surface d'onde de $1 / 5$ à $1 / 4$ de frange pour les meilleurs, pour des angles d'incidence Littrow de $74^{\circ}$; l'intensité dı premier "ghost " est de $10^{-3}$ dans le visible. Actuellement, on en est au stade où l'on s'occupe des erreurs locales et de İa forme des sillons, défauts comparables à ceux étudiés par MM. Lyot et Frənçon pour les lentilles de coronographe.

En résumé l'instrument de $\mathrm{P}$. Connes ne sera pas limité par la qualité des réseaux.

M. Migeotte. - Les réseaux de 6 pouces $(15 \mathrm{~cm})$ du National Physical Laboratory sont également bien exempts de ghosts. 